\title{
FBG sensor strain performance analysis using optisystem software tools
}

\author{
Muhammad Imaduddin Mohd Razi, Mohd Rashidi Che Beson, Saidatul Norlyana Azemi, \\ Syed Alwee Aljunid \\ School of Computer and Communication Engineering, Universiti Malaysia Perlis, Malaysia
}

\begin{tabular}{|c|c|}
\hline Article Info & ABSTRACT \\
\hline Article history: & Fibre Bragg Grating is an optical fibre that widely use as sensor to sense \\
\hline Received Sep 27, 2018 & $\begin{array}{l}\text { strain, temperature and pressure. The advantages of FBG sensor are it will } \\
\text { not affected by electromagnetic interference which can improve the accuracy }\end{array}$ \\
\hline Revised Nov 26, 2018 & of the result and also can be wearable sensor. In this paper, Optisystem \\
\hline Accepted Jan 27, 2019 & $\begin{array}{l}\text { software tools are used to analyse the strain performance of FBG sensor for } \\
\text { monitoring knee joint movement in rehabilitation process. Single mode FBG }\end{array}$ \\
\hline Keywords: & $\begin{array}{l}\text { with original wavelength of } 1550 \mathrm{~nm} \text { are used to get the accurate result } \\
\text { because of less attenuation and absorption factor. This project also analysed }\end{array}$ \\
\hline Fibre bragg grating & the wavelength shifted when strain experienced by FBG change. \\
\hline Medical & $\begin{array}{l}\text { The accuracy of sensor and method of delivering the result are taken into the } \\
\text { account. The interrogator are used to display the wavelength shifted by the }\end{array}$ \\
\hline Sensor & $\begin{array}{l}\text { FBG when there is change in strain experienced by FBG. All of the proposed } \\
\text { designed are tested by using simulation software and the wavelength shifted } \\
\text { in the simulation are compared to the theoretical value that has been } \\
\text { calculated in this project. The development of this system expected to } \\
\text { improve the rehabilitation process towards a healthy life. }\end{array}$ \\
\hline
\end{tabular}

Copyright $(2019$ Institute of Advanced Engineering and Science. All rights reserved.

\section{Corresponding Author:}

Muhammad Imaduddin Mohd Razi,

School of Computer and Communication Engineering,

Universiti Malaysia Perlis,

Pauh Putra Campus, 02600 Arau, Perlis, Malaysia.

Email: imaduddinph94@gmail.com

\section{INTRODUCTION}

The requirement for monitoring body kinematics has become a vast developing area in engineering field. Because of the huge advantages, it is now applied to the field of medicine. Universities, highperformance sport centers and health-care institutions have been investing in developing ways to measure and evaluate accurately the way the human body moves for endless purposes [1]. At the same time, the usage of fiber optic sensors (FOSs) is receiving large acknowledgement as a replacement to conventional electrical and mechanical sensors for monitoring of mechanical and thermal parameters. The main principle in development of monitoring body kinematics is Fiber Bragg Grating also abbreviated as FBG. FBG is a passive and discrete optical component at a particular spot in an optical fiber. It is a type of optical sensor which reflects a wavelength of light that shifts in response to differences in temperature or strain. FBG are made by exposing the core of a single-mode fiber to a periodic pattern of intense ultraviolet light. The exposure creates a constant increase in the refractive index of the fiber's core, thus producing a fixed index modulation called a grating.

\subsection{Theory and Working Principle}

Fiber Bragg Grating (FBG) sensors are a significant finding particularly in smart sensor applications. FBG sensors have presented a huge potential for applications in the field of biomechanics and 
rehabilitation engineering. This is due to its benefits such as its tiny size, bio friendly and resistance to electromagnetic interference (EMI), chemical inertness and multiplexing ability [2]. Currently, FBGs are extensively used for monitor and measuring numerous parameters such as temperature, strain, humidity, pressure, force and radiation dose. The problem of typical sensor which is electronic sensor is presence of high electromagnetic and high voltage. The solution to overcome this problem is by using optical fiber sensor because it is made of dielectric material sand so it is considerable to locate them close or even upon a high latent conductor and they don't actually need electrical power at the sensor area. FBG can be operated and applied for in vivo measurement and placed for lengthy period observing because it is harmless to human physique [3].

Other than that, FBG are also better than conventional gauges in vivo applications because the risk of infection is smaller. Bragg grating is created when the germanium-doped fiber which is photosensitive type was subjected to the UV light. The amount of the change depends on the intensity and duration of the exposure as well. The principle operation of FBG sensor is by observing the wavelength shifting of the output signal with the differences in the measuring such as strain or temperature of the light reflected from an FBG [4]. Periodic variation $\Lambda$ of the FBG and the refractive index influenced the reflected wavelength which is called Bragg wavelength $(\lambda B)[5]$. The general equation of the FBG is

$$
\lambda B=2 n_{e f f} \Lambda
$$

FBG sensor systems must consist of an optical source that continuously interrogates the reflection spectrum. It also must consist a detection module that monitor the shifts in the peak reflectivity versus wavelength. From these component, it can measure wavelength shifts that result directly from the physical changes.

If external or thermal perturbations is exposed to the FBG sensor, the reflected band will be shifted. FBG sensor is very responsive to strain and temperature. The axial strain influences the FBG reaction specifically through the strain-optic impact which incites an adjustment in the optical fiber effective index, and through the pressure and development changes in dividing of the periodic variation, $\Lambda$. The temperature increase $\Delta \mathrm{T}$ influences the grating period and the refractive index, thus lead to an adjustment in $\lambda \mathrm{B}$. Definition of the refractive index and the impact of strain and temperature on grating period is

$$
\Delta \lambda_{B}=\left[\lambda \frac{\delta n}{\delta l}+n \frac{\delta \Lambda}{\delta l}\right] \Delta l+2\left[\Lambda \frac{\delta n}{\delta T}+n \frac{\delta \Lambda}{\delta T}\right] \Delta T
$$

$\Delta \mathrm{l}$ is differences of Fiber Bragg Grating length affected by strain while $\Delta \mathrm{T}$ is the change of temperature [4]. Optical Spectrum Analyzer (OSA) is generally used to identify the Bragg wavelength for experiment in laboratories while for outside experiment, specialized interrogators are used.

There are available different types of sensors from optical fiber. It can be classified into three classes, the intrinsic type, extrinsic type and the hybrid type. The optical properties of intrinsic type are sensitive to temperature and strain [7]. For the transmission of information to a long distance location, extrinsic type is the best option [8].

In addition, for the transmission of light through the optical fiber for transference into electricity on a long distance optical receiver, the hybrid type is the most compatible choice [9]. From the earlier stated categories, over the past 20 years, the intrinsic sensors have been learnt and applied thoroughly [10]. The intrinsic element in Fiber Bragg grating sensor influenced sensor performance. Because of the photosensitivity optical fiber, ultraviolet (UV) light beams can listed the grating. Besides that, inbuilt selfreferencing and multiplexing capability are also the features of FBG.

Basically, the FBG is a periodic variant of the refraction index along the fiber axis. The function of the structure is to act as a reject-band filter, reflecting back the spectral component, $\Delta \lambda B$ [nm], which contents the Bragg condition and transmitting the remaining components. Giving below is the equation of the wavelength shift, $\Delta \lambda B[\mathrm{~nm}]$, of a FBG sensor subject to a physical disturbance [11]:

$$
\frac{\Delta \lambda_{B}}{\lambda_{B}}=\left(1-\rho_{e}\right) \Delta \varepsilon+(\alpha+\xi) \Delta T
$$

where $p e, \Delta, \alpha, £$, and $\Delta \mathrm{T}$ are the effective photo elastic constant, the axial strain, the thermal expansion, the thermal optic coefficient and the temperature shifts, respectively. The ratio wavelength shift, $\Delta \lambda B$ to wavelength, $\lambda B$ in the (1) expresses the strain effect on an optical fiber. A change in the grating spacing and the strain-optic induced change in the refractive index correspond by the strain effect.

The second term of the expression is correlated with the temperature sensing. It dilates or contracts as the FBG is subjected to temperature variation thus modifying the grating pitch. Flexible sensing structure. 
Increasing the sensitivity of the sensor will give a precise measurement of the knee joint movement. This was performed by appropriately choosing the material that can transduce perfectly the real movement. The conversion of the movements toward the embedded sensor would be enable if the area to cover the knee is sufficient. A wide rectangular design was selected to improve cover both the extension movements and the flexion.

Furthermore, it offers the required area of contact to be interpreted by the sensing area and let the light to transport without any unexpected corners that would block the communication with the monitoring stage. The advantages and features of this foil include the stretch ability, the capability and the flexibility [1]. All these feature will maintained an effective bonding between the optical fiber and the substrate. The main material is polyvinyl chloride (PVC) to ensure the stimulus transfer and the bonding. Moreover, during fabrication, its shape and size are totally modifiable.

\subsection{Related Works}

Figure 1 a) the green ellipses is the flexible knee with pressure buttons, For b), the flexible knee band is connected with sensing part and c), the red circle indicate PVC foil with the embedded FBG signaled.

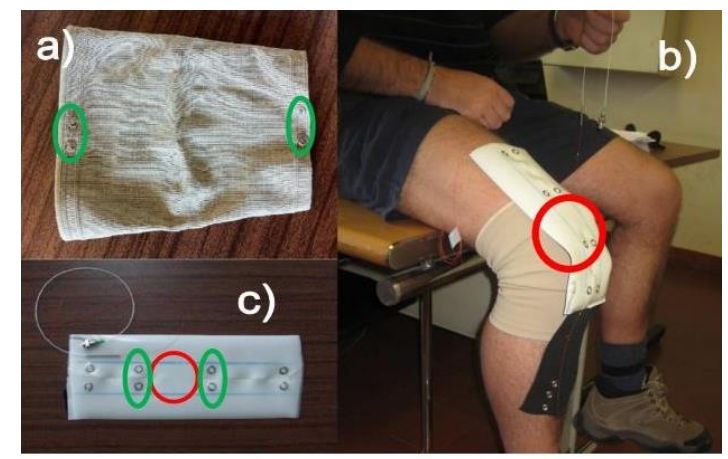

Figure 1. FBG in PVC foil [1]

Authors [1] has developed a PVC foil based on FBG sensor that can be used to measure the body kinematics. This system is wearable system that can monitor maximum knee movement, flexibility and all the changes during a full period of human movement. A single Fiber Bragg Grating as located at the center of the knee joint with the rectangular structure that combined with 3 layer of elastic PVC materials. All the movement connected with a full steps, the position and swing stages, are obviously identifiable in the acquired waveform permitting evaluation between various results gained in several circumstances.

The advantage of this system is user friendly because the sensing system can simply attached or take out from the flexible knee band via using small metallic pressure button to confirm FBG is only sensing the extension and the flexion as the subject steps around. Besides that, the system also resistance to electromagnetic interference. Thus, it can be used in wet environment and inside water without harm.

Disadvantage of the system is the dimensions of the flexible knee band and the method of connecting the foil with embedded sensor can decrease the quick oscillations of the waveform. Moreover, if the speed of subject movement is high, the waveform will become noisier compared to the slow moving subject.

Figure 2 shows a) to capture image movement of the hand in real time on a PC, a hand shaped PVC foil contains 14 FBGs in the upper surface of the glove. In b)left, the reaction of FBG sensor when step of opening and closing hand while in (right), a graph that represent comparison of measured and real angles to clarify system precision. For monitoring both posture and hand gesture of stroke patients' brain, author in [12] had developed a wearable FBG sensing glove. This allow the angle measurement between the finger phalanxes.

A single optical fiber that contain 14 FBGs are located in al layout that curvilinear and fixed in the middle layer of an elastic PVC foil that are hand shaped to ensure the FBG are located at 14 phalanx joints respectively in a single hand. The system display sinusoid like response. The major benefit of this wearable sensor is the measurement made on wavelength as a replacement of optical power. So, the instabilities of the optical source will make the system insensitive to external issues. Besides, the system also can be stable for long term as the polymer foil enlarge resistance to the sensing element without affecting the performance of 
sensing elements. Moreover, the glove can simply worn without limiting the movement of the hand. The disadvantages of the system is wrinkles may be appear at the glove due to flexibility of the hand.
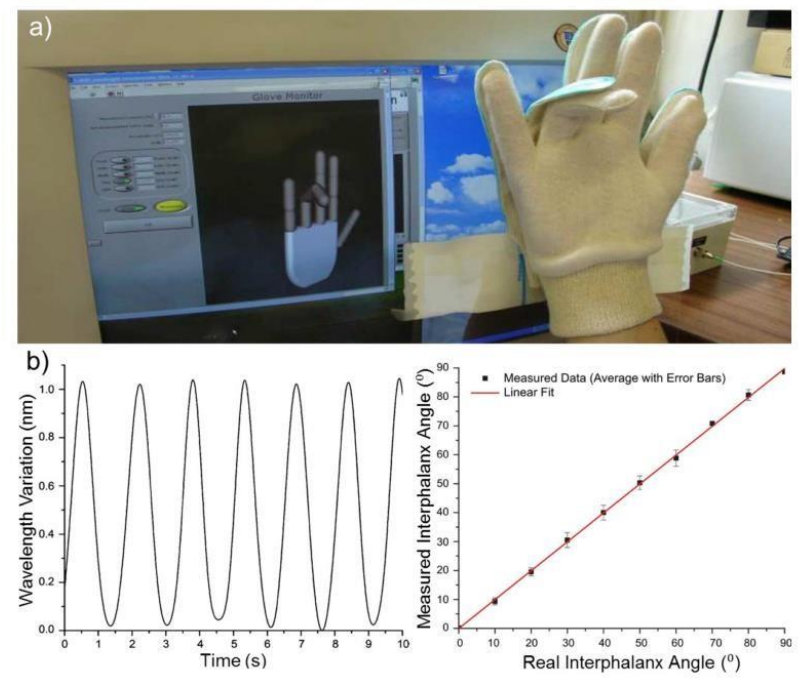

Figure 2. FBG Layout for Monitoring Hand Posture [12]

Figure 3 shows a) Experiment setup during the project and b) graph comparison between untreated and decalcified bones. Author in [13] proposed the consequence of decalcification on strain reaction of a goat tibia. It was investigated in vitro using Fiber Bragg Grating (FBG) sensing technique. In order to analyze the strength of bone sample, the straining actions need to be simulated as closely as possible.

There are four types of straining actions. However, bending test was chosen in this experiment. Three-point bending technique was used to apply tension to two similar bone samples from the same goat and one was partly decalcified. The Bragg wavelength also were recorded during the experiment. As a result, the strain caused for the same pressure increased along with larger degree of decalcification. Also an abrupt increase took place after $2 \mathrm{~g}$ of calcium loss, signifying the beginning of damage. This proved that FBG sensor technology can be used effectively to clarify alterations in same due to different degrees of decalcification.

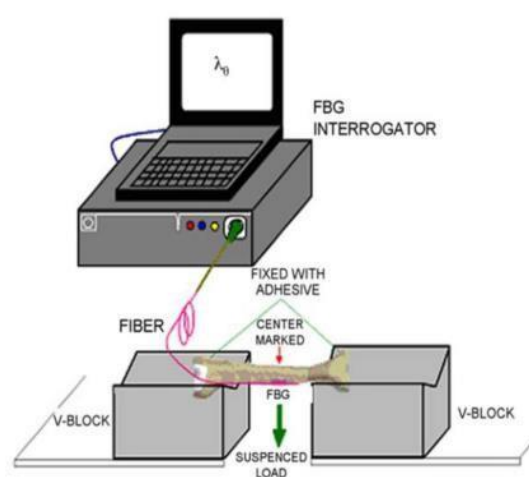

a

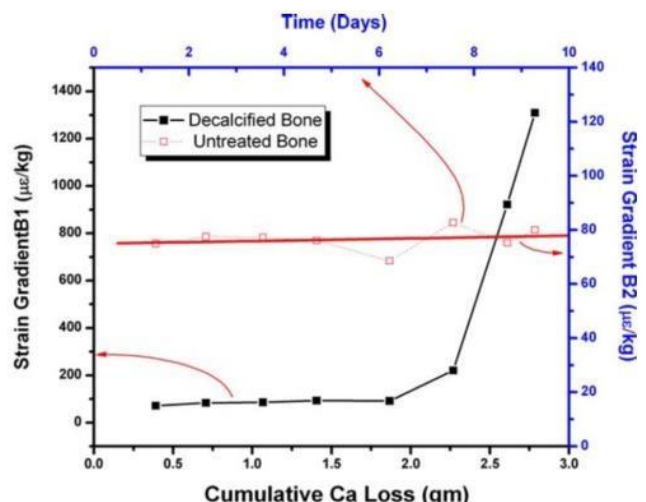

b

Figure 3. Experimental setup and comparison graph

\section{RESEARCH METHOD}

The focus of this project is to analyze the performance of the sensor when the sensor is applied with certain value of strain. Hence, the value of strain applied can be used to analyze the maximum strain that can 
be applied on the Fiber Bragg Grating. Optical Spectrum Analyzer that will be used in this project simulation to display the wavelength shifting. The research was all carried out using simulation software, Optisystem version 14.2.

\subsection{Strain Sensing Equation}

$$
\lambda B=2 n_{e f f} \Lambda
$$

$\mathrm{B}$ is Bragg wavelength is the refractive index and $\Lambda$ is periodic variation. By using the above equation, expand the equation to get the shift in the Bragg grating center directly to the strain change is set as

$$
\left.\Delta \lambda=\left[\left(1-\rho_{e}\right) \times \varepsilon\right)+\left(\left(\alpha_{\lambda}+\alpha_{n}\right) \times \Delta T\right)\right] \lambda_{0}
$$

where $\Delta \lambda$ is the wavelength shift, is the initial wavelength, is the thermal expansion coefficient, is the thermal optic coefficient, $\Delta \mathrm{T}$ is the temperature change and is the strain experienced by grating. pe is strain optic coefficient which can be defined as

$$
\left(\frac{n^{2}}{2}\right)\left[p_{12}-v\left(p_{11}+p_{12}\right)\right]
$$

where $\mathrm{n}$ is the refractive index of fiber, is the strain tensor component and $\mathrm{v}$ is the Poisson ratio.

\subsection{Block Diagram of Optisystem Design}

Figure 4 shows block diagram of optisystem design.

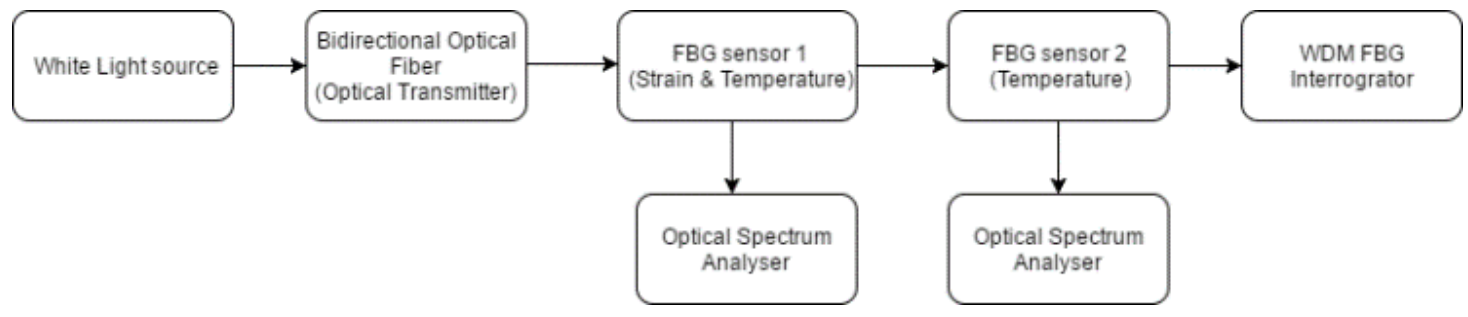

Figure 4. Block diagram of optisystem design

\subsection{Design and Parameter}

Figure 5 shows simulation design.

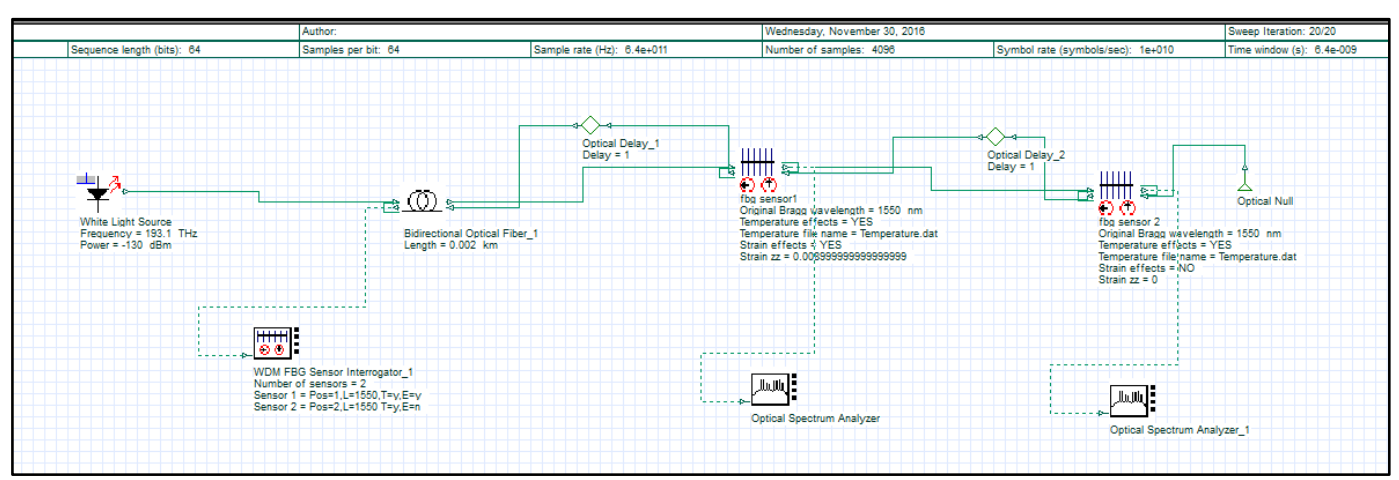

Figure 5. Simulation design

The design will contain one white light source, two Fiber Bragg Grating sensor, two optical delay, one optical null, bidirectional optical fiber, two optical spectrum analyzer and one WDM FBG sensor interrogator. For the FBG sensor, FBG sensor 1 will sense temperature and strain while FBG sensor 2 is for 
measuring temperature only. WDM FBG Sensor Interrogator is for show the wavelength shifted by the reflected spectrum of the two FBG sensor. Strain were set at the parameter from 0.005 to 0.01 .Optical delay were used to avoid situation which the simulation has not perform because a component cannot find signals at the input port and the system will be deadlocked. Other than that, all parameter in the Optisystem were using default setting.

\section{RESULTS AND ANALYSIS}

Simulation result are the result of six simulation by using the Optisystem 14.2 simulation, which is by using six different value of strain to analyze the performance of FBG sensor and wavelength shifting. Theoretical calculation was performed by using formula of wavelength shifting of FBG sensor for strain and temperature. Calculation of wavelength shifting (theoretical) as shows in Table 1 and Wavelength shifting simulation result shows in Table 2. Figure 6 shows wavelength shifting versus strain experienced by FBG (theoretical). Figure 7 shows wavelength shifting versus strain experienced by FBG (simulation). Figure 8 shows comparison between the theoretical and simulation wavelength shifting.

Table 1. Calculation of Wavelength Shifting (Theoretical)

\begin{tabular}{|c|c|c|c|}
\hline $\begin{array}{c}\text { Initial Wavelength, } \boldsymbol{\lambda}_{\mathbf{0}} \\
(\mathrm{nm})\end{array}$ & $\begin{array}{c}\text { Temperature Change, } \\
{ }^{\circ} \mathrm{C}\end{array}$ & $\begin{array}{c}\text { Strain } \\
\text { Experienced By } \\
\text { FBG }\end{array}$ & $\begin{array}{c}\text { Wavelength } \\
\text { Shifted, } \Delta \boldsymbol{\lambda} \\
(\mu \mathrm{m})\end{array}$ \\
\hline 1550 & 2 & 0.005 & 0.01010 \\
\hline 1550 & 2 & 0.006 & 0.01134 \\
\hline 1550 & 2 & 0.007 & 0.01258 \\
\hline 1550 & 2 & 0.008 & 0.01382 \\
\hline 1550 & 2 & 0.009 & 0.01506 \\
\hline 1550 & 2 & 0.01 & 0.01630 \\
\hline
\end{tabular}

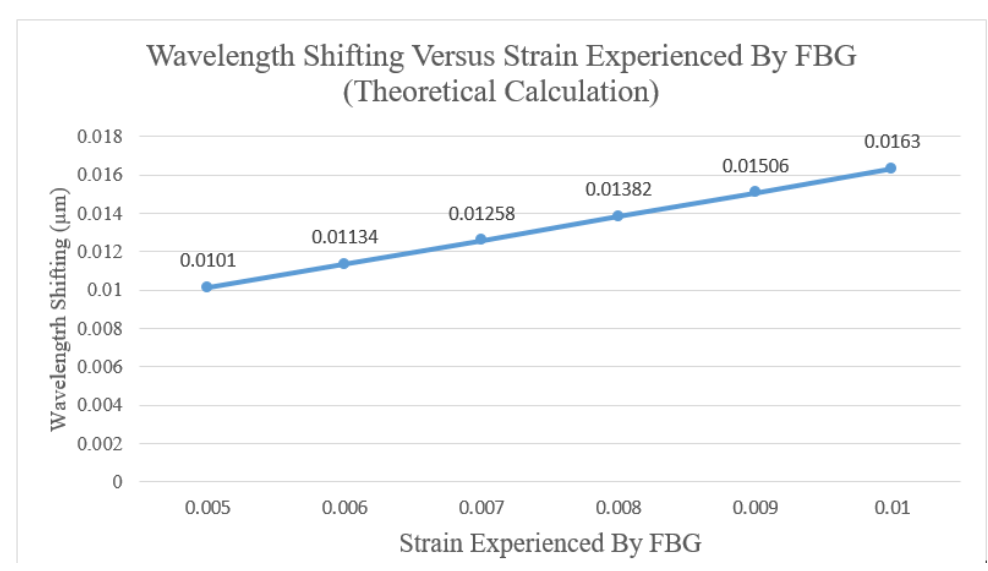

Figure 6. Wavelength Shifting Versus Strain Experienced by FBG (theoretical)

Table 2. Wavelength Shifting Simulation Result

\begin{tabular}{|c|c|c|c|}
\hline $\begin{array}{c}\text { Initial } \\
\text { Wavelength, } \lambda_{\mathbf{0}} \\
(\mathrm{nm})\end{array}$ & $\begin{array}{c}\text { Temperature Change, } \\
{ }^{\circ} \mathrm{C}\end{array}$ & $\begin{array}{c}\text { Strain } \\
\text { Experienced By } \\
\text { FBG }\end{array}$ & $\begin{array}{c}\text { Wavelength } \\
\text { Shifted, } \Delta \lambda \\
(\mu \mathrm{m})\end{array}$ \\
\hline 1550 & 2 & 0.005 & 0.001 \\
\hline 1550 & 2 & 0.006 & 0.0072 \\
\hline 1550 & 2 & 0.007 & 0.0085 \\
\hline 1550 & 2 & 0.008 & 0.0097 \\
\hline 1550 & 2 & 0.009 & 0.0109 \\
\hline 1550 & 2 & 0.01 & 0.012 \\
\hline
\end{tabular}




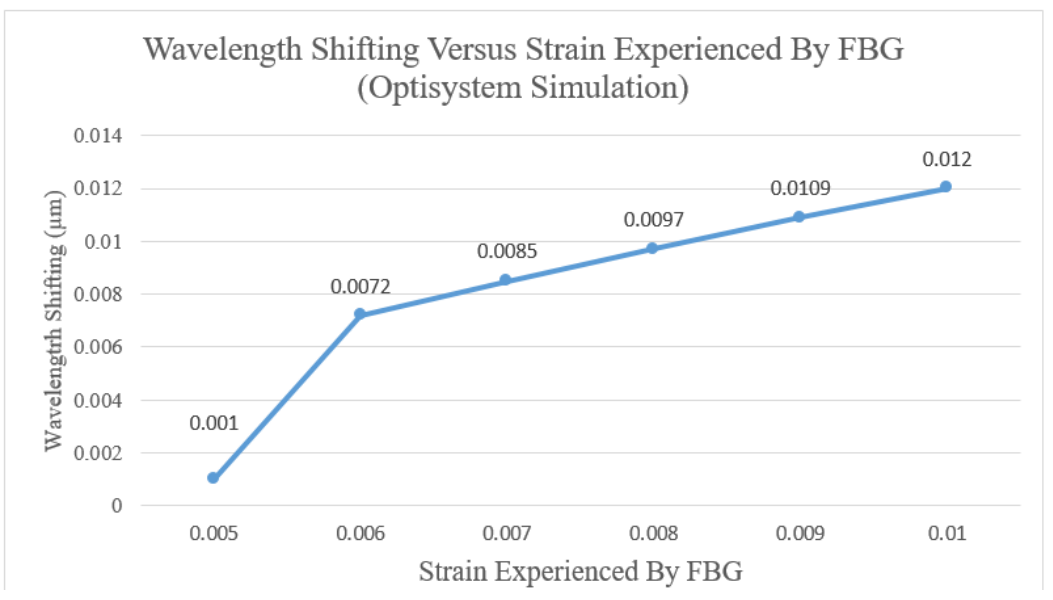

Figure 7. Wavelength Shifting Versus Strain Experienced by FBG (simulation)

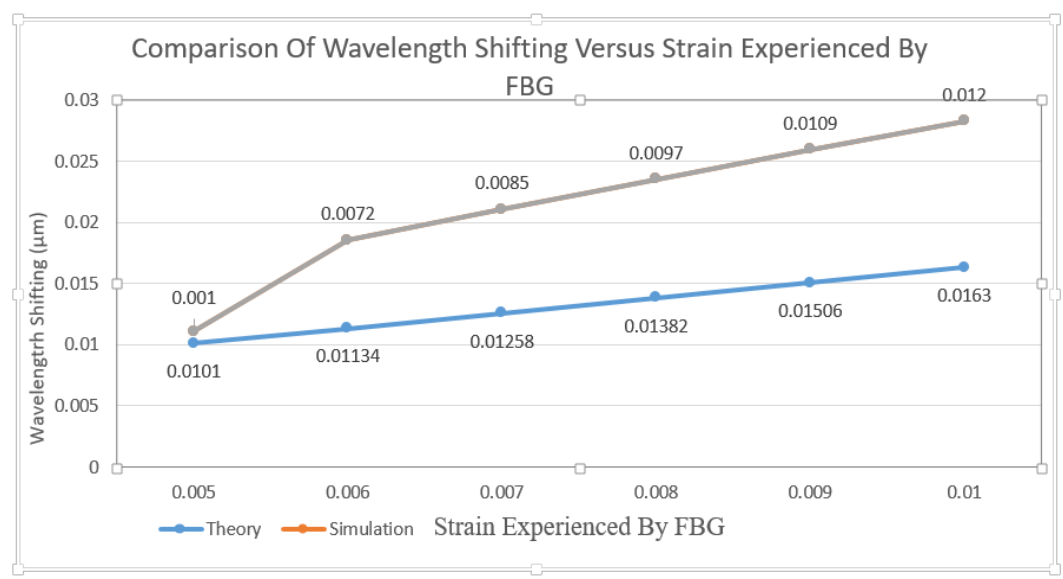

Figure 8. Comparison between the Theoretical And Simulation Wavelength Shifting

From the graph, the theory and simulation value were not the same due to the design parameter set in the simulation software. Besides, there were also bidirectional optical fiber in the simulation design that may affect the value of wavelength shifting for the simulation. Simulation result is greater than theoretical because there is more wavelength shifting.

\section{CONCLUSION}

The FBG design in the simulation of Optisystem 14.2 was tested by using 6 different strain value that experienced by the two FBG sensor. Based on the result from simulation, wavelength shifting will easily been analyzed. Besides, there are only slightly difference between theoretical value and simulation value.

\subsection{Limitation and Constrain}

This project was mainly about analyzing the performance of FBG sensor that can be used for medical application. There are several problem been faced during the development of this project. One of the problem is the FBG sensor is not tested in human joint. This cause the result from the simulation was not accurate enough because it was not tested on the human joint. Besides, the wavelength shifting and strain that experienced by FBG was not based of human kinetic movement. The FBG sensor in Optisystem also has strain value limitation that cause the wavelength were not shifting when the value of strain were changed.

\subsection{Future works}

For the future work, it is suggested to use the real FBG sensor and tested to the human based on the human kinetic movement. So, the result accuracy will be improve and the main purpose of this project which 
is to develop the sensor for monitoring the joint movement during rehabilitation process can be achieved. Another suggestion is to use another software that can simulate the FBG sensor based on human kinetic movement so that the wavelength shifting that been measured is based on the human joint movement.

\section{REFERENCES}

[1] R. P. Rocha, A. F. Silva, J. P. Carmo, and J. H. Correia, "FBG in PVC foils for monitoring the knee joint movement during the rehabilitation process," Proc. Annu. Int. Conf. IEEE Eng. Med. Biol. Soc. EMBS, pp. $458-461,2011$.

[2] C. R. Dennison, P. M. Wild, D. R. Wilson, and P. A. Cripton, "A minimally invasive in-fiber Bragg grating sensor for intervertebral disc pressure measurements," Meas. Sci. Technol., vol. 19, no. 8, p. 85201, 2008.

[3] S. J. Mihailov, "Fiber bragg grating sensors for harsh environments," Sensors, vol. 12, no. 2. pp. 1898-1918, 2012.

[4] E. Al-Fakih, N. A. A. Osman, and F. R. M. Adikan, "The use of fiber bragg grating sensors in biomechanics and rehabilitation applications: The state-of-the-art and ongoing research topics," Sensors (Switzerland), vol. 12, no. 10, pp. 12890-12926, 2012.

[5] Z. Fang, K. K.Chin, R. Qu, and H. Cai, Fundamentals of Optical Fiber Sensors Wiley Series in Microwave and Optical Engineering. 2012.

[6] C. Yan, E. Ferraris, T. Geernaert, F. Berghmans, and D. Reynaerts, "Development of flexible pressure sensing polymer foils based on embedded fibre Bragg grating sensors," Procedia Eng., vol. 5, pp. 272-275, 2010.

[7] M. Boerkamp, D. W. Lamb, and P. G. Lye, "Using an intrinsic, exposed core, optical fibre sensor to quantify chemical scale formation," J. Phys. Conf. Ser., vol. 76, p. 12016, 2007.

[8] P. C. Beard and T. N. Mills, "Extrinsic optical fibre ultrasound sensor using a thin polymer film as a low finesse Fabry-Perot interferometer,” Appl. Opt., vol. 34, no. 4, pp. 663-675, 1996.

[9] S. K. Yao and C. K. Asawa, "Fiber Optical Intensity Sensors," IEEE J. Sel. Areas Commun., vol. 1, no. 3, pp. 562-575, 1983.

[10] B. Lee, "Review of the present status of optical fiber sensors," Opt. Fiber Technol., vol. 9, no. 2, pp. 57-79, 2003.

[11] C. L. Wei et al., "A fiber Bragg grating sensor system for train axle counting," IEEE Sens. J., vol. 10, no. 12, pp. 1905-1912, 2010.

[12] A F. Da Silva, A. F. Gonçalves, P. M. Mendes, and J. H. Correia, "FBG sensing glove for monitoring hand posture,” IEEE Sens. J., vol. 11, no. 10, pp. 2442-2448, 2011.

[13] V. Mishra et al., "Fiber Bragg grating sensor for monitoring bone decalcification," Orthop. Traumatol. Surg. Res., vol. 96, no. 6, pp. 646-651, 2010.

[14] a F. Silva, J. P. Carmo, P. M. Mendes, and J. H. Correia, "Simultaneous cardiac and respiratory frequency measurement based on a single fiber Bragg grating sensor," Meas. Sci. Technol., vol. 22, no. 7, p. 75801, 2011.

[15] N. Din Keraf et al., "An optimal cardinality of wavelength/time incoherent OCDMA system using 2-D hybrid FCC-MDW code," in 2014 2nd International Conference on Electronic Design, ICED 2014, 2011, pp. 356-361.

[16] C. B. M. Rashidi, S. A. Aljunid, F. Ghani, M. S. Anuar, and H. A. Fadhil, "Code length optimization using Flexible Cross Correlation (FCC) code in OCDMA networks," in ICP 2012 - 3rd International Conference on Photonics 2012, Proceedings, 2012, pp. 355-359.

\section{BIOGRAPHIES OF AUTHORS}

\begin{tabular}{|l|l|} 
Muhammad Imaduddin Mohd Razi received the B.Eng. in Computer Network Engineering from \\
University Malaysia Perlis (UniMAP), Malaysia. He is currently completing the M.Sc. degree in \\
Communication Enginering at University Malaysia Perlis (UniMAP), Malaysia. His research \\
interest include optical sensing and optical communication.
\end{tabular}




\begin{tabular}{|l|l|}
\hline & $\begin{array}{l}\text { Saidatul Norlyana Azemi received her M.Sc degree in Communication Engineering from } \\
\text { Universiti Malaysia Perlis (UniMAP), Malaysia and Ph.D degree in Electric \& Electronic } \\
\text { Engineering from, Royal Melbourne Institute of Technology (RMIT) University, Australia. She } \\
\text { is currently working as senior lecturer in School of Computer and Communication Engineering, } \\
\text { Universiti Malaysia Perlis (UniMAP), Malaysia. }\end{array}$ \\
\hline & $\begin{array}{l}\text { Syed Alwee Aljunid Syed Junid received his B.Eng in Computer System and Communication, } \\
\text { Universiti Putra Malaysia, UPM and his Ph.D in Communication And Network Engineering, } \\
\text { also from Universiti Putra Malaysia, UPM. He also received his B.T.E.C Higher National } \\
\text { Diploma in Engineering U.K. His expertise include optical communication system. }\end{array}$ \\
\hline
\end{tabular}

